\title{
Representation of Integer Positive Number as A Sum of Natural Summands
}

\author{
Vadim N. Romanov \\ St.-Petersburg, Russia
}

\section{ABSTRACT}

In this paper the problem of representation of integer positive number as a sum of natural terms is considered. The new approach to calculation of number of representations is offered. Results of calculations for numbers from 1 to 500 are given. Dependence of partial contributions to total sum of number of representations is investigated. Application of results is discussed.

Keywords and phrases: Theory of numbers, representation of integer positive number as a sum of natural terms, number of representations, distribution of partial contributions.

\section{INTRODUCTION. STATEMENT OF THE PROBLEM AND GENERAL RELATIONS}

The problem of representation of integer positive number as a sum of the natural summands (terms) has a long story. M. Hall in his book [1] provides an overview of works on this subject and gives representations calculated for the numbers from 1 to 100 . However the problem is not solved yet. The purpose of the paper is to study some new aspects of this problem, and to obtain an efficient algorithm for the calculation of the number of representations. The problem is formulated in the following form. A positive integer $n$ is given. We want to find the number of its different representations as a sum of the natural terms. Designate $C(n)$ - number of representations of $n$ as a sum of terms and let $n=n$ - identity representation. Let's call the number of representations of $n$ as a sum of terms including the identical representation the proper representation $C_{S}(n)$, and without identical representation - the improper representation $C_{N S}(n)$. It is obvious $C_{N S}(n)=C_{S}(n)-1$. Hereinafter we mean the proper representation and omit the subscript that does not bring misunderstandings. We give the algorithm of calculation of $C(n)$ for an arbitrary $n$, and deduce a recurrent relation that allows to reduce the dimensionality of the problem. For arbitrary natural $n$ we have

$C(n)=\pi_{1}(n)+\pi_{2}(n)+\pi_{3}(n)+\ldots+\pi_{n-1}(n)+\pi_{n}(n)$

where $\pi_{1}(n)=1-$ number of representations of $n$ as a sum of units: $n=1+1+\ldots+1 ; \pi_{2}(n)-$ number of representations of $n$ as a sum of units and at least one number two; $\pi_{3}(n)$ - number of representations of $n$ as a sum of units, deuces and at least one number three, etc. $\pi_{n-1}(n)$ - number of representations of $n$ as a sum of units, deuces etc. and at least one number $(n-1) ; \pi_{n}(n)=1$ number of identical representations. Hereinafter we call value $\pi_{k}(n)$ the partial contributions. To reduce the dimension of the problem we use the following obvious ratios

$\pi_{1}(n)=\pi_{1}(n-1)=1$,

$\pi_{2}(n)=\pi_{1}(n-2)+\pi_{2}(n-2)$,

$\pi_{3}(n)=\pi_{1}(n-3)+\pi_{2}(n-3)+\pi_{3}(n-3)$ etc.

In the general case we have for arbitrary $l$ $\pi_{l}(n)=\pi_{1}(n-l)+\pi_{2}(n-l)+\ldots+\pi_{l}(n-l)$,

(2)

where $\pi_{l}(n)$ - number of representations of $n$ as a sum at least one $l$ and numbers smaller $l$, $\pi_{1}(n-l)$ - number of representations of $n-l$ as a sum of units: $\pi_{1}(n-l)=1 . \pi_{2}(n-l)-$ number of representations of $n-l$ as a sum of units and at least one number two; etc. $\pi_{l}(n-l)$ - number of representations of $n-l$ as a sum at least one number $l$ and numbers smaller $l$. The following relation is deduced from (2)

$\pi_{n-l}(n)=\pi_{1}(l)+\pi_{2}(l)+\ldots+\pi_{n-l}(l)$.

It is clear that for calculations it is better to use (2) at $n-l \leq l$ and (3) at $n-l>l$. Equations (2), (3) provide an opportunity to reduce dimensionality and 
perform calculations for the smallest of two numbers $l$ or $n-l$, i.e. to use representations for numbers, that are not more than $n / 2$. Note also that part of the summands in (2), (3) can vanish. Generally speaking for the calculation $\pi_{l}(n)$ are important only the difference $n-l$ and number $l$. For two numbers $n_{1}, n_{2}$ and the other two $l_{1}, l_{2}$ such that $l_{1}<n_{1}, \quad l_{2}<n_{2}, \quad n_{1}-l_{1}<l_{1}, \quad n_{2}-l_{2}<l_{2}$, $n_{1}-l_{1}=n_{2}-l_{2}$ we have the ratio

$\pi_{l_{1}}\left(n_{1}\right)=\pi_{l_{2}}\left(n_{2}\right)$.

Consider the example of calculation. Let $n=6$. We write the representation (1) for this case $C(6)=\pi_{1}(6)+\pi_{2}(6)+\pi_{3}(6)+\ldots+\pi_{5}(6)+\pi_{6}(6)$ (5)

where in accordance with (2), (3)

$$
\begin{aligned}
& \pi_{1}(6)=1, \\
& \pi_{2}(6)=\pi_{1}(4)+\pi_{2}(4)=\pi_{1}(4)+\pi_{1}(2)+\pi_{2}(2)=3 \\
& \pi_{3}(6)=\pi_{1}(3)+\pi_{2}(3)+\pi_{3}(3)=3 ; \\
& \pi_{4}(6)=\pi_{1}(2)+\pi_{2}(2)=2 ; \\
& \pi_{5}(6)=\pi_{1}(1)=1 ; \pi_{6}(6)=1 .
\end{aligned}
$$

From (5) we obtain $C(6)=11$. When $n$ increases this way of calculation is difficult.

\section{ALGORITHM OF CALCULATION}

To facilitate the calculations we write expressions for the partial contributions in (2). In particular, for $\pi_{2}(n-l)$ this is easy to do. Using successively (2), we obtain

$\pi_{2}(n-l)=\pi_{1}(n-l-2)+\pi_{1}(n-l-4)+\ldots+\pi_{1}(n-l-2 k)+\pi_{2}(n-l-2 k)=k+1$ (6)

where $k=\left[\frac{n-l-2}{2}\right]-$ the whole part of number in brackets. For $\pi_{3}(n-l)$ the ratio has the form

$$
\pi_{3}(n-l)=1+\sum_{k=1}^{t}\left[\frac{n-l-3 k}{2}\right]+\sum_{k=1}^{t_{1}} 1,
$$

where $t=\left[\frac{n-l-2}{3}\right], t_{1}=\left[\frac{n-l-3}{3}\right]$.

For calculation of other components it is more convenient to use recurrent relations deduced from (2) - (4) and (6), (7). Put in (6), (7) $l=0$. It is easy to obtain the following expressions for the partial contributions $\pi_{1}(n), \ldots, \pi_{n}(n)$ for arbitrary $n$ : $\pi_{1}(n)=1 . \pi_{n}(n)=1$.

$\pi_{2}(n)=1+\left[\frac{n-2}{2}\right]$.

$\pi_{3}(n)=1+\sum_{k=1}^{\left[\frac{n-2}{3}\right]}\left[\frac{n-3 k}{2}\right]+\sum_{1}^{\left[\frac{n-3}{3}\right]} 1$.

$\pi_{4}(n)=\pi_{3}(n-1) \quad$ when $0 \leq\left[\frac{n-4}{4}\right]<1$, i.e.

$4 \leq n<8$

or

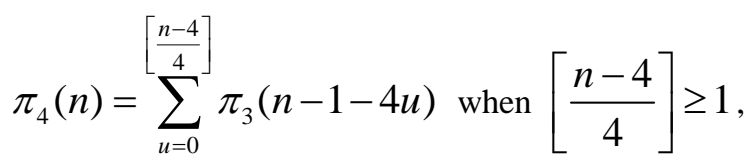

i.e. $n \geq 8$.

In the general case for arbitrary $k \geq 4$ we have $\pi_{k}(n)=\pi_{k-1}(n-1)$ when $0 \leq\left[\frac{n-k}{k}\right]<1$, i.e.

$k \leq n<2 k$

or

$\pi_{k}(n)=\sum_{u=0}^{\left[\frac{n-k}{k}\right]} \pi_{k-1}(n-1-k u)$ when

$\left[\frac{n-k}{k}\right] \geq 1$, i.e. $n \geq 2 k$.

$\pi_{n-1}(n)=\pi_{n-2}(n-1)$ when $0 \leq\left[\frac{1}{n-1}\right]<1$, i.e.

$n \geq 3$.

$\pi_{n-1}(n)=1$ when $n \geq 2$.

(14)

For given number $n$ the ratio (12a) is performed if $k \leq n / 2$; the ratio (12) is true for $k>n / 2$ if $n$ is even and for $k>(n+1) / 2$ if $n$ is odd. From (2) it is easy to obtain the following relationship $\pi_{k}(n)=\pi_{k-1}(n-1)+\pi_{k}(n-k)$.

If we take into account the obtained relations, the algorithm of calculation of partial contributions has the form

1. $\pi_{1}(n)=1$ is defined for arbitrary $n$.

2. $\pi_{2}(n), \pi_{3}(n)$ are calculated according to (9), (10).

3. $\pi_{k}(n)$ are calculated according to (12) for appropriate conditions.

4. $\pi_{k}(n)$ are calculated according to (15) for appropriate conditions. 
Position 2 can be used for additional verification of the results. In fact for the calculation of all partial contributions it is sufficient to have the value $\pi_{1}(n)=\operatorname{const}(n)=1$ and to use the relation (15) or (12) depending on the ratio of $n$ and $k$ to accelerate the calculations. When partial contributions are determined the values $C_{S}(n)$ are obtained by their summation for each $n$. Thus the obtained relations allow to determine the number of representations of arbitrary $n$ as a sum of natural summands. The calculations were carried out in Excel according to (15) and (12) that immediately allows to extend result obtained for the selected initial values $n$ and $k$ for all $n$ and $k$. The sample results of calculation are given in tables 1, 2 .

\section{STUDY OF PARTIAL CONTRIBUTIONS AND DISCUSSION OF RESULTS}

In addition we investigated the dependence of the maximum contributions $\pi_{k}(n)$ to $C_{S}(n)$. The results are given in table 3 . In table 4 the values of partial contributions $\pi_{k}(n)$ for some $n$ are given. The dependence of partial contributions $\pi_{k}(n)$ to the value $C_{S}(n)$ on $k$ for certain $n$ coincides in the form with the Poisson distribution. If we divide the value of the contribution $\pi_{k}(n)$ by $C_{S}(n)$, we obtain value of probability given by this distribution. In table 5 the relative values of partial contributions for certain $n$ are given. For each $n$ the envelope of the dependence of relative partial contributions on $k$ is approximated by a set of functions of the form $y=a x^{b} \exp \left(c x^{d}\right)+g$ with parameters $a, b, c, d, g$ dependent on $n$, where $c<$ $0, b>1$. The parameter $g$ can be put equal to zero if $n \geq 50$. The variable $x$ changes from 0 to $\infty$, although we are interested in the interval from 1 to $n$. The dependence of the maximum of relative partial contributions on $k$ is also approximated by specified set of functions with $c<0, b<0$. From table 5 it follows that the distribution "spreads" and simultaneously the share of partial contributions the value of which exceeds 0.01 decreases with increasing $n$. So, for $n=10$ the half-width of the envelope is 3 , and the share of contributions $100 \%$ (all contributions have the value more than 0.01); for $n=25$ the half-width is 7 and the share of contributions $60 \%$; for $n=50$ the corresponding values are equal to 15 and $26 \%$; for $n=150$ values are 19 and 20\% respectively; for $n=200: 23$ and 16.5\%; for $n=250: 25$ and 14\% (the round-off values). Analysis of the results in table 3 shows that the maximum contribution to the value $C_{S}(n)$ given by partial contributions $\pi_{k}(n)$ with the number $k$ is not very strongly dependent on $n$ and when we change $n$ from 4 to 1000 value $k$ changes from 2 to 81 . Thus the distribution of partial contributions has a significant asymmetry which increases with increasing $n$. Each $k$ corresponds to an interval of values $n$ which increases slowly with increasing $n$. This trend is continued beyond the values from table 3 . In particular, for $n=513-527$ the value of the maximum is at $k=53$, for $n=528$ 542 at $k=54$, for $n=543-558$ at $k=55$, for $n=559$ 573 at $k=56$, for $n=574-589$ at $k=57$, for $n=590$ 605 at $k=58$, for $n=606-621$ at $k=59$, for $n=622-$ 637 at $k=60$, for $n=638-653$ at $k=61$, for $n=654$ 670 at $k=62$, for $n=671-687$ at $k=63$, for $n=688$ 703 at $k=64$ for $n=704-720$ at $k=65$, for $n=721$ 738 at $k=66$, for $n=739-755$ at $k=67$, for $n=756$ 772 at $k=68$, for $n=773-790$ at $k=69$, for $n=791$ 807 at $k=70$, for $n=808-825$ at $k=71$, for $n=826$ 843 at $k=72$, for $n=844-862$ at $k=73$ for $n=863$ 880 at $k=74, n=881-898$ at $k=75$, for $n=899-917$ at $k=76$, for $n=918-936$ at $k=77$, for $n=937-954$ at $k=78$, for $n=955-974$ at $k=79$, for $n=975-993$ at $k=80$, for $n=994-1012$ at $k=81$. Function $k_{\max }(n)$ changes stepwise and the speed of its growth is 0.2 for $n=1-50 ; 0.14$ for $n=50-100 ; 0.1$ for $n=100-250$; 0.08 for $n=250-400$; 0.06 for $n=$ 400-1000.

\section{APPLICATION OF RESULTS}

Consider some applications of obtained results. The values of $C_{S}(n)$ given in tables 1,2 can be used in differential topology to calculate groups of cobordism [2]. The problem of representation is also associated with problems of number theory in particular, the binary Goldbach's conjecture and the hypothesis of Legendre [3]. The results of table 4 give the opportunity for technical application of obtained relationships. Each number $n$ corresponds to the definite sequence of partial sums $\pi_{k}(n)$. Therefore such sequences can be used in digital filtering and digital coding. This allows to increase significantly the stability of the code to various failures and errors. So, for number $n=250$ the error is 0,004 and in its representation as the sequence of partial contributions the error is about $10^{-15}\left(C_{S}(250)=230793554364681\right)$.

The obtained results can be also used for analysis (description, modeling) of complex systems. Consider the example. Suppose that there is a chaos consisting of $n$ elements. At some point, if there are conditions, the elements begin randomly to connect (join together) one, two, three, etc. forming 
different ordered structures which can be considered as parts (subsystems) of the overall system. As the dimension of these ordered structures, as well as, the variety of such structures is one of the main characteristics of complexity, the total number $C_{S}(n)$ of such ordered structures (subsystems) shows the complexity of the resulting system. From the results of calculation of $C_{S}(n)$ in tables 1,2 it follows that for $n$ from 6 to 32 can be formed only small (simple) systems, for $n$ between 33 and 60 complex systems are formed, for $n$ from 61 to 406 ultra-complex systems are formed, for $n$ from 407 and more super-systems are formed. We can conclude (see table 3) that from initial chaos containing $n$ elements most likely the formation of ordered structures with a maximum contribution to $C_{S}(n)$. For close values of $n$ there is a dead zone for $k$, which is understandable, because the change of the contributions is small as compared with their absolute value. From table 5 it follows that equally with a structure corresponding to $k_{\max }(n)$ the structures can be also formed which probability of occurrence is close to the maximum $k_{\max }(n)$. The number of such structures increases with increasing $n$, however their relative share decreases, which indicates a clear orientation of process. If we assume that the formation of structures depends on some parameter, e.g. temperature, with the change of temperature the complexity of the structures and their diversity determined by the value of $n$ increase. Thus from the chaos can be formed complex ordered structures characterized by significant diversity; this process has a definite tendency and it has a stability and adaptability to the conditions, as well as, the formed structures. From results in table 3 it follows that the process of formation of ordered structures is long, since the probability of their formation is small though finite. We can regard this example as a model for the origin and development of life.

Table 1: The values of $C_{S}(n)$ for some $n$ from 1 to 260

\begin{tabular}{|c|c|c|c|}
\hline$n$ & $C_{S}(n)$ & $n$ & $C_{S}(n)$ \\
\hline 1 & 1 & 110 & 607163746 \\
\hline 6 & 11 & 120 & 1844349560 \\
\hline $\mathbf{1 0}$ & $\mathbf{4 2}$ & 130 & 5371315400 \\
\hline 20 & 627 & 140 & 15065878135 \\
\hline 30 & 5604 & $\mathbf{1 5 0}$ & $\mathbf{4 0 8 5 3 2 3 5 3 1 3}$ \\
\hline 32 & 8349 & 160 & 107438159466 \\
\hline 33 & 10143 & 170 & 274768617130 \\
\hline 40 & 37338 & 180 & 684957390936 \\
\hline $\mathbf{5 0}$ & $\mathbf{2 0 4 2 2 6}$ & 190 & 1667727404093 \\
\hline 60 & 966467 & $\mathbf{2 0 0}$ & $\mathbf{3 9 7 2 9 9 9 0 2 9 3 8 8}$ \\
\hline 61 & 1121505 & 210 & 9275102575355 \\
\hline 70 & 4087968 & 220 & 21248279009367 \\
\hline 80 & 15796476 & 230 & 47826239745920 \\
\hline 90 & 56634173 & 240 & 105882246722733 \\
\hline $\mathbf{1 0 0}$ & $\mathbf{1 9 0 5 6 9 2 9 2}$ & $\mathbf{2 5 0}$ & $\mathbf{2 3 0 7 9 3 5 5 4 3 6 4 6 8 1}$ \\
\hline 101 & 214481126 & 260 & 495741934760846 \\
\hline & & & \\
\hline
\end{tabular}

Table 2: The values of $C_{S}(n)$ for some $n$ from 270 to 500

\begin{tabular}{|c|c|c|c|}
\hline$n$ & $C_{S}(n)$ & $n$ & $C_{S}(n)$ \\
\hline 270 & 1050197489931120 & $\mathbf{4 0 0}$ & $\begin{array}{c}\mathbf{6 7 2 7 0 9 0 0 5 1 7 4 1 0 4} \\
* \mathbf{1 0 0 0 0}\end{array}$ \\
\hline 280 & 2195786311682520 & 406 & $\begin{array}{c}972551251374203 \\
* 10000\end{array}$ \\
\hline 290 & 4534253126900890 & 407 & $\begin{array}{c}103390972671239 \\
* 100000\end{array}$ \\
\hline $\mathbf{3 0 0}$ & $\mathbf{9 2 5 3 0 8 2 9 3 6 7 2 3 6 0 0}$ & 410 & $\begin{array}{c}124166774031512 \\
* 100000\end{array}$ \\
\hline 310 & 18671488299600400 & 420 & 227552902165800 \\
& & & 4100000 \\
\hline 320 & 37274405776748100 & 430 & 414157392071024 \\
\hline
\end{tabular}




\begin{tabular}{|c|c|c|c|}
\hline & & & $* 100000$ \\
\hline 330 & 73653287861850200 & 440 & $\begin{array}{c}748782484194708 \\
* 100000\end{array}$ \\
\hline 340 & $\begin{array}{c}144117936527874 \\
* 1000\end{array}$ & $\mathbf{4 5 0}$ & $\begin{array}{c}\mathbf{1 3 4 5 0 8 1 8 8 0 0 1 5 7 3} \\
* \mathbf{1 0 0 0 0 0 0}\end{array}$ \\
\hline $\mathbf{3 5 0}$ & $\mathbf{2 7 9 3 6 3 3 2 8 4 8 3 7 0 2}$ & 460 & $\begin{array}{c}240123655613925 \\
* \mathbf{1 0 0 0}\end{array}$ \\
& \begin{tabular}{c}
$* 1000000$ \\
\hline 360
\end{tabular} & 470 & $\begin{array}{c}426088638015652 \\
* 1000000\end{array}$ \\
\hline 370 & $\begin{array}{c}102214122836735 \\
* 10000\end{array}$ & 480 & $\begin{array}{c}751666004194992 \\
* 1000000\end{array}$ \\
\hline 380 & $\begin{array}{c}193065607235047 \\
* 10000\end{array}$ & 490 & $\begin{array}{c}131852040161227 \\
* 10000000\end{array}$ \\
\hline 390 & $\begin{array}{c}361771276386760 \\
* 10000\end{array}$ & $\mathbf{5 0 0}$ & $\begin{array}{c}\mathbf{2 3 0 0 1 6 5 0 3 2 5 7 4 3 3} \\
* \mathbf{1 0 0 0 0 0 0 0}\end{array}$ \\
\hline
\end{tabular}

Note. Since $n=270$ the values of $C_{S}(n)$ are given with rounding to $10^{-15}$.

Table 3: The values of $k$ for the maximum contribution $\pi_{k}(n)$ and the relative values of the maximum contribution $\pi_{k}(n) / C_{S}(n)$ for $n$ from 1 to 500

\begin{tabular}{|c|c|c|c|c|c|}
\hline$n$ & $k$ & $\pi_{k}(n) / C_{S}(n)$ & $n$ & $k$ & $\pi_{k}(n) / C_{S}(n)$ \\
\hline 1 & 1 & 1 & $152-161$ & 24 & $0,046-0,045$ \\
\hline 2 & 1,2 & 0,5 & $162-171$ & 25 & $0,045-0,044$ \\
\hline 3 & $1,2,3$ & $1 / 3$ & $172-181$ & 26 & $0,043-0,042$ \\
\hline 4 & 2 & 0,4 & $182-192$ & 27 & $0,042-0,041$ \\
\hline $5-6$ & 2,3 & $0,3-0,27$ & $193-202$ & 28 & $0,041-0,040$ \\
\hline 7 & 3 & 0,27 & $203-213$ & 29 & $0,039-0,038$ \\
\hline 8 & 3,4 & 0,23 & $214-224$ & 30 & $0,038-0,037$ \\
\hline 9 & 3 & 0,23 & $225-235$ & 31 & $0,037-0,036$ \\
\hline $10-12$ & 4 & $0,21-0,19$ & $236-247$ & 32 & $0,036-0,035$ \\
\hline $13-14$ & 4,5 & $0,18-0,17$ & $248-258$ & 33 & $0,035-0,0345$ \\
\hline $15-17$ & 5 & $0.17-0,16$ & $259-270$ & 34 & $0,0346-0,034$ \\
\hline $18-22$ & 6 & $0,15-0,14$ & $271-282$ & 35 & $0,034-0,033$ \\
\hline $23-28$ & 7 & $0,13-0,12$ & $283-294$ & 36 & $0,033-0,032$ \\
\hline $29-34$ & 8 & $0,11-0,105$ & $295-306$ & 37 & $0,032-0,0315$ \\
\hline $35-39$ & 9 & $0,104-0,098$ & $307-319$ & 38 & $0,0315-0,031$ \\
\hline $40-46$ & 10 & $0,096-0,089$ & $320-331$ & 39 & $0,031-0,030$ \\
\hline $47-53$ & 11 & $0,088-0,082$ & $332-344$ & 40 & $0,030-0,0296$ \\
\hline $54-59$ & 12 & $0,082-0,078$ & $345-357$ & 41 & $0,0296-0,029$ \\
\hline $60-67$ & 13 & $0,077-0,072$ & $358-371$ & 42 & $0,029-0,0284$ \\
\hline $68-74$ & 14 & $0,072-0,069$ & $372-384$ & 43 & $0,0284-0,028$ \\
\hline $75-82$ & 15 & $0,068-0,065$ & $385-397$ & 44 & $0,028-0,0274$ \\
\hline $83-89$ & 16 & $0,064-0,062$ & $398-411$ & 45 & $0,0274-0,027$ \\
\hline $90-98$ & 17 & $0,061-0,059$ & $412-425$ & 46 & $0,027-0,0264$ \\
\hline $99-106$ & 18 & $0,059-0,056$ & $426-439$ & 47 & $0,0264-0,026$ \\
\hline $107-115$ & 19 & $0,056-0,054$ & $440-453$ & 48 & $0,026-0,0255$ \\
\hline $116-124$ & 20 & $0,054-0,052$ & $454-468$ & 49 & $0,0255-0,025$ \\
\hline $125-133$ & 21 & $0,051-0,050$ & $469-482$ & 50 & $0,025-0,0247$ \\
\hline $134-142$ & 22 & $0,049-0,048$ & $483-497$ & 51 & $0,0246-0,024$ \\
\hline $143-151$ & 23 & $0,048-0,046$ & $498-512$ & 52 & $0,024-0,0238$ \\
\hline
\end{tabular}

Note. The relative values are given with rounding. 
Table 4: The value of partial contributions $\pi_{k}(n)$ for some $n$

\begin{tabular}{|c|c|}
\hline$n$ & $\pi_{k}(n)$ \\
\hline 1 & 1 \\
\hline 10 & $1,5,8, \underline{\mathbf{9}}, 7,5,3,2,1,1$ \\
\hline 25 & $1,12,52,120,192,235, \mathbf{2 4 8}, 230,201,164,131,100,77,56,42,30,22,15,11,7,5,3,2,1,1$ \\
\hline 50 & $\begin{array}{l}1,25,208,920,2611,5427,8946,12450,15224,16928, \underline{\mathbf{1 7 4 7 5}}, 17084,15988,14499,12801,11098, \\
9459,7976,6647,5507,4520,3699,3003,2434,1958,1575,1255,1002,792,627,490,385,297,231, \\
176,135,101,77,56,42,30,22,15,11,7,5,3,2,1,1\end{array}$ \\
\hline 100 & 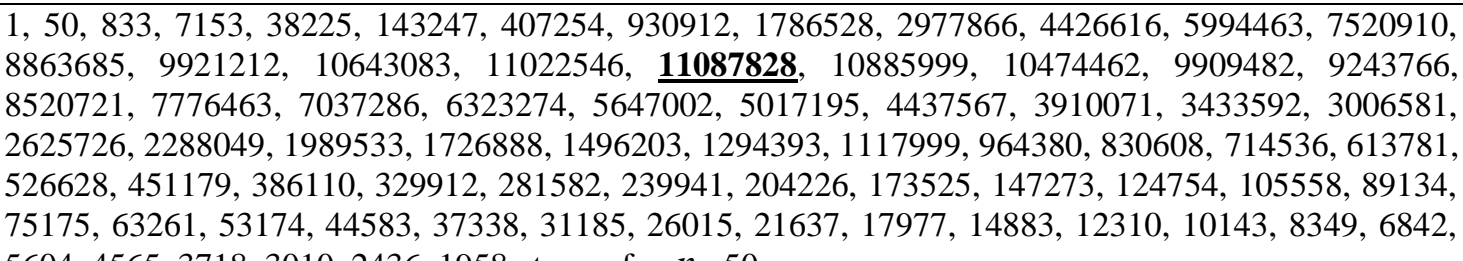 \\
\hline
\end{tabular}
$5604,4565,3718,3010,2436,1958$ etc., as for $n=50$

$1501,75,1875,23906,187572,1015691,4097732,13026135,34040565,75611815,146622950$, 253686437, 398700683, 577696317, 781305581, 996568049, 1209282272, 140628590, 1576846549, 1713879071, 1813657483, 1875641076, 1901740434, 1895596591, 1861842424, 1805546155, $1731734353,1645120184,1549889427,1449643115,1347348172,1245393246,1145613858$, 1049387370, 957678579, 871134698, 790125385, 714823012, 645228970, 581235788, 522640439, 469190374, 420586425, 376516904, 336653352, 300675693, 268264132, 239117954, 212945213, 189477547, 168458928, 149657501, 132854604, 117854413, 104473449, 92548950, 81929021, 72479844, 64076888, 56611148, 49981183, 44098813, 38881910, 34259456, 30165270, 26542448, 23337785, 20505882, 18004132, 15796379, 13848605, 12132145, 10619856, 9289089, 8118264, 7089500, 6185689, 5392783, 4697205, 4087968, 3554345, 3087735, 2679689, 2323520, 2012558, 1741630, 1505499, 1300156, 1121505, 966467, 831820, 715220, 614154, 526823, 451276, 386155, $329931,281589,239943,204226$ etc., as for $n=100$

200 1, 100, 3333, 56389, 583464, 4132096, 21591496, 87914929, 290766028, 807151588, 1930988902, 4068707635, 7688392411, 13228519842, 20992222004, 31061127403, 43254681491, 57144118454, $72112062630,87438760128, \quad 102392668573, \quad 116308613844, \quad 128642751969, \quad 139002300504$, $147151784574, \quad 153002189595, \quad 156588477188, \quad \underline{\mathbf{1 5 8 0 4 2 2 3 1 8 4 3}}, \quad 157562992547, \quad 155392337462$, $151791444014,147023829564,141342227857,134980139819,128146377172,121022840700$, 113763863853, 106497447819, 99326965343, 92333833284, 85579990891, 79110819757, 72957552004, 67139866109, 61667868042, 56544168977, 51765357145, 47323553668, 43207424083, 39403290219, 35895772746, 32668559740, 29704776023, 26987499334, 24499940379, 22225781156, 20149228887, 18255234620, 16529467501, 14958453606, 13529504579, 12230804801, 11051315499, 9980830549, 9009870095, 8129716789, 7332308815, 6610264380, 5956778650, 5365642518, 4831146028, 4348093735, 3911716861, 3517686778, 3162035607, 2841169553, 2551797606, 2290944586, 2055888160, 1844171590, 1653547895, 1481992987, 1327656113, 1188872777, 1064121426, 952035923, 851367332, 760996393, 679899697, 607161659, 541945028, 483502160, 431149016, 384276141, 342325612, 304801320, 271248931, 241265372, 214481124, 190569292, 169229875, 150198136, 133230930, 118114304, 104651419, 92669720, 82010177, 72533807, 64112359, 56634173, 49995925, 44108109, 38887673, 34262962, 30167357, 26543660, 23338469, 20506255, 18004327, 15796476, 13848650, 12132164, 10619863, 9289091, 8118264 etc., as for $n=150$

$2501,125,5208,109809,1410797,12342963, \quad 79192479, \quad 393157376, \quad 1573234010,5240306955$, 14913616956, 37052772644, 81826474002, 163087911253, 297200495920, 500751117265, 787620248236, 1166142118732, 1637068720885, 2192796266004, 2817943187204, 3491044933605, 4186925982162, 4879267901753, 5542956439698, 6155935255066, 6700433968275, 7163573972280, $7537431109655,7818690201309,8008025707702,8109342822208, \underline{\mathbf{8 1 2 8 9 7 6 4 4 4 2 8 7}}, 8074929570885$, 7956192621917, 7782175369928, 7562252794944, 7305427257644, 7020089114421, 6713866994803, 6393545176509, 6065038537649, 5733404877102, 5402888470851, 5076979170730, 4758484892967, 4449606070852, 4152013088989, 3866918486279, 3595146988743, 3337197329538, 3093299988224, 2863466161337, 2647532516564, 2445197837743, 2256056125188, 2079622741308, 1915357941952, 1762684686988, 1621004758944, 1489710281669, 1368194326786, 1255857870654, 1152116441015, 1056403873148, 968176201278, 886913245776, 812120639284, 743330003171, 680099753443, 
622014399317, 568684587082, 519745891469, 474858406882, 433705285321, 395992084206, 361445211812, 329811171459, 300855017393, 274359578827, 250123990790, 227962949046, 207705347412, 189193594613, 172282373092, 156838028946, 142737458535, 129867575006,

$118124317268, \quad 107412193480, \quad 97643404649, \quad 88737458105, \quad 80620398260, \quad 73224482905$, 66487509215, 60352547438, 54767351459, 49684140987, 45059088668, 40852143568, 37026584253, 33548878862, 30388295652, 27516792708, 24908680039, 22540533675, 20390901601, 18440239357, 16670653737, 15065855110, 13610935153, 12292332535, 11097639253, 10015578174, 9035833989, $8149039483,7346628828,6620830516,5964539309,5371315303,4835271825,4351078581$, 3913864288, 3519222690, 3163127352, 2841940500, 2552338241, 2291320912, 2056148051, $1844349560,1653668665,1482074143,1327710076,1188908248,1064144451,952050665$, 851376628, 761002156, 679903203, 607163746, 541946240, 483502844, 431149389, 384276336, 342325709, 304801365, 271248950, 241265379, 214481126, 190569292, 169229875, 150198136, 133230930, 118114304, 104651419, 92669720, 82010177, 72533807, 64112359, 56634173, 49995925, 44108109, 38887673, 34262962, 30167357, 26543660, 23338469, 20506255, 18004327, 15796476, 13848650, 12132164, 10619863, 9289091, 8118264, 7089500, 6185689, 5392783, 4697205, 4087968, 3554345, 3087735, 2679689, 2323520, 2012558, 1741630, 1505499, 1300156, 1121505, 966467, 831820, 715220, 614154, 526823, 451276, 386155, 329931, 281589, 239943, 204226, 173525, 147273, 124754, 105558, 89134, 75175, 63261, 53174, 44583, 37338, 31185, 26015, 21637, 17977, 14883, $12310,10143,8349,6842,5604,4565,3718,3010,2436,1958,1575,1255,1002,792,627,490,385$, $297,231,176,135,101,77,56,42,30,22,15,11,7,5,3,2,1,1$

Note. Underlined and in bold the maximum values of contributions.

Table 5: The relative value of partial contributions $\pi_{k}(n) / C_{S}(n)$ for some $n$

\begin{tabular}{|c|c|}
\hline$n$ & $\pi_{k}(n) / C_{S}(n)$ \\
\hline 1 & 1 \\
\hline 10 & $\overline{0}, 0238,0,119,0,191, \underline{\mathbf{0 , 2 1 4}}, 0,167,0,119,0,0715,0,0476,0,0238,0,0238$ \\
\hline 25 & $\begin{array}{l}0,00051,0,00613,0,0266,0,0613,0,098,0,12, \underline{\mathbf{0 , 1 2 6 5}}, 0,117,0,103,0,0837,0,067,0,051,0,0395, \\
0,0286,0,0214,0,0153,0,0112,0,00766,0,00562,0,00358,0,00256,0,00153,0,00102,0,00051,0,00051\end{array}$ \\
\hline 50 & $\begin{array}{l}0,49 \cdot 10^{-5}, 1,23 \cdot 10^{-4}, 1,02 \cdot 10^{-3}, 0,45 \cdot 10^{-2}, 0,0128,0,0266,0,0438,0,061,0,0745,0,0829, \underline{\mathbf{0 , 0 8 5 7}}, 0,0838, \\
0,0785,0,0711,0,0627,0,0545,0,0464,0,0391,0,0326,0,028,0,0222,0,0181,0,0147,0,0119,0,96 \cdot 10^{-2}, \\
0,772 \cdot 10^{-2}, 0,615 \cdot 10^{-2}, 0,49 \cdot 10^{-2}, 0,388 \cdot 10^{-2}, 0,306 \cdot 10^{-2}, 0,204 \cdot 10^{-2}, 0,189 \cdot 10^{-2}, 0,145 \cdot 10^{-2}, 0,113 \cdot 10^{-2}, \\
0,0862 \cdot 10^{-2}, 0,0662 \cdot 10^{-2}, 0,0495 \cdot 10^{-2}, \ldots, 0,49 \cdot 10^{-5}\end{array}$ \\
\hline 100 & $\begin{array}{l}0,525 \cdot 10^{-8}, 0,262 \cdot 10^{-6}, 0,432 \cdot 10^{-5}, 0,376 \cdot 10^{-4}, 0,201 \cdot 10^{-3}, 0,75 \cdot 10^{-3}, 0,214 \cdot 10^{-2}, 0,489 \cdot 10^{-2}, 0,94 \cdot 10^{-2}, \\
1,51 \cdot 10^{-2}, 2,32 \cdot 10^{-2}, 3,15 \cdot 10^{-2}, 3,95 \cdot 10^{-2}, 4,65 \cdot 10^{-2}, 5,21 \cdot 10^{-2}, 5,6 \cdot 10^{-2}, 5,78 \cdot 10^{-2}, \mathbf{0 , 0 5 8 3}, 0,0572,0,0552, \\
0,052,0,0485,0,0447,0,0409,0,037,0,0332,0,0297,0,0264,0,0233,0,0205,0,018,0,0158,0,0138, \\
0,012,0,01045,0,0091,0,00788,0,00678,0,00585,0,00505,0,00436,0,00375,0,00322,0,00277, \\
0,00237,0,00202,0,00173,0,00147,0,00126,0,00107,0,000912,0,000771,0,000656,0,000554, \ldots, \\
0,525 \cdot 10^{-8}\end{array}$ \\
\hline 150 & $\begin{array}{l}0,245 \cdot 10^{-10}, 1,84 \cdot 10^{-9}, 0,46 \cdot 10^{-7}, 0,585 \cdot 10^{-6}, 0,46 \cdot 10^{-5}, 0,249 \cdot 10^{-4}, 1 \cdot 10^{-4}, 3,18 \cdot 10^{-4}, 0,833 \cdot 10^{-3}, 0,185 \cdot 10^{-2}, \\
0,36 \cdot 10^{-2}, 0,622 \cdot 10^{-2}, 0,977 \cdot 10^{-2}, 1,415 \cdot 10^{-2}, 1,915 \cdot 10^{-2}, 2,44 \cdot 10^{-2}, 2,96 \cdot 10^{-2}, 3,45 \cdot 10^{-2}, 3,87 \cdot 10^{-2}, 4,19 \cdot 10^{-2}, \\
4,43 \cdot 10^{-2}, 4,6 \cdot 10^{-2}, \mathbf{0 , 0 4 6 6}, 4,65 \cdot 10^{-2}, 4,56 \cdot 10^{-2}, 4,42 \cdot 10^{-2}, 4,24 \cdot 10^{-2}, 4,03 \cdot 10^{-2}, 3,8 \cdot 10^{-2}, 3,55 \cdot 10^{-2}, 3,31 \cdot 10^{-2}, \\
3,05 \cdot 10^{-2}, 2,8 \cdot 10^{-2}, 2,57 \cdot 10^{-2}, 2,35 \cdot 10^{-2}, 2,14 \cdot 10^{-2}, 1,94 \cdot 10^{-2}, 1,75 \cdot 10^{-2}, 1,58 \cdot 10^{-2}, 1,425 \cdot 10^{-2}, 1,28 \cdot 10^{-2}, \\
1,15 \cdot 10^{-2}, 0,0103,0,00921,0,00825,0,00737,0,00656,0,00585,0,00522,0,00463,0,00411,0,00366, \\
0,00326,0,00289,0,00256,0,00229,0,00201,0,00178,0,00157,0,00139,0,00123,0,00108,0,000921, \\
0,000825,0,000737,0,000657, \ldots, 0,245 \cdot 10^{-10}\end{array}$ \\
\hline 200 & $\begin{array}{l}0,252 \cdot 10^{-12}, 0,252 \cdot 10^{-10}, 0,631 \cdot 10^{-9}, 1,42 \cdot 10^{-8}, 1,47 \cdot 10^{-7}, 1,04 \cdot 10^{-6}, 0,544 \cdot 10^{-5}, 2,22 \cdot 10^{-5}, 0,734 \cdot 10^{-4}, \\
2,03 \cdot 10^{-4}, 0,486 \cdot 10^{-3}, 1,025 \cdot 10^{-3}, 1,94 \cdot 10^{-3}, 0,332 \cdot 10^{-2}, 0,529 \cdot 10^{-2}, 0,784 \cdot 10^{-2}, 0,0109,0,0144,0,0182, \\
0,022,0,0257,0,0292,0,0325,0,0348,0,037,0,0386,0,0394, \underline{\mathbf{0 , 0 3 9 9}}, 0,0398,0,039,0,0383,0,037, \\
0,0355,0,034,0,0322,0,0305,0,0287,0,0267,0,025,0,0232,0,0216,0,0199,0,0184,0,0169,0,0155, \\
0,0142,0,0129,0,0119,0,0109,0,00993,0,00904,0,00824,0,00748,0,0068,0,00617,0,00559,0,00506, \\
0,0046,0,00416,0,00377,0,0034,0,00307,0,00278,0,00251,0,00227,0,00204,0,00185,0,00166, \\
0,0015,0,00135,0,00122,0,001095,0,0985 \cdot 10^{-2}, 0,0886 \cdot 10^{-2}, 0,0796 \cdot 10^{-2}, 0,0715 \cdot 10^{-2}, \ldots, 0,252 \cdot 10^{-12}\end{array}$ \\
\hline 250 & $0,434 \cdot 10^{-14}, 0,521 \cdot 10^{-12}, 2,26 \cdot 10^{-11}, 0,476 \cdot 10^{-9}, 0,611 \cdot 10^{-8}, 0,533 \cdot 10^{-7}, 3,42 \cdot 10^{-7}, 1,7 \cdot 10^{-6}, 0,68 \cdot 10^{-5}$, \\
\hline
\end{tabular}


$2,27 \cdot 10^{-5}, 0,581 \cdot 10^{-4}, 1,6 \cdot 10^{-4}, 3,54 \cdot 10^{-4}, 0,706 \cdot 10^{-3}, 1,29 \cdot 10^{-3}, 2,17 \cdot 10^{-3}, 0,00341,0,00506,0,0071$, $0,00949,0,01215,0,0151,0,0181,0,0211,0,024,0,0267,0,029,0,031,0,0326,0,0338,0,0346,0,0351$, $\underline{\mathbf{0 , 0 3 5 2}}, 0,0349,0,0344,0,0337,0,0327,0,0316,0,0304,0,029,0,0277,0,0262,0,0248,0,0234,0,022$, $0,0206,0,0193,0,018,0,01675,0,0156,0,0147,0,0134,0,0124,0,0115,0,0106,0,00975,0,009,0,0083$, $0,00763,0,00702,0,00645,0,00594,0,00544,0,00498,0,00457,0,00419,0,00384,0,00352,0,00322$, $0,00294,0,00269,0,00246,0,00225,0,00206,0,00188,0,00171,0,00156,0,00143,0,0013,0,00119$, $0,00108,0,0984 \cdot 10^{-2}, 0,0901 \cdot 10^{-2}, 0,0819 \cdot 10^{-2}, 0,0745 \cdot 10^{-2}, 0,068 \cdot 10^{-2}$, $0,0619 \cdot 10^{-2}, 0,00563 \cdot 10^{-2}, \ldots, 0,434 \cdot 10^{-14}$

Note. Underlined and in bold the maximum values of contributions. For each $n$ to the left of the maximum are given all the contributions, and to the right of the maximum only the contributions with values not less than 0.001 (with rounding) and the value of the last contribution.

\section{REFERENCES}

[1]. Hall M. Combination theory - Moscow: Mir, 1970 (in Russian).

[2]. Hirsh M. Differential topology. - Moscow: Mir, 1979 (in Russian).

[3]. Romanov V. N., Study of fundamental problems of number theory. Saint-Petersburg, Publishing company "Asterion", 2015(in Russian). 